\title{
The gas and dust coma of Comet C/1999 H1 (Lee)^
}

\author{
L.-M. Lara ${ }^{1}$, R. Rodrigo ${ }^{1}$, G. P. Tozzi ${ }^{2}$, H. Boehnhardt ${ }^{3}$, and P. Leisy ${ }^{4}$ \\ 1 Instituto de Astrofísica de Andalucía, CSIC, PO Box 3004, 18080 Granada, Spain \\ e-mail: rodrigo@iaa.es \\ 2 INAF, Osservatorio Astrofisico di Arcetri, Largo E. Fermi 5, 50125 Firenze, Italy \\ e-mail: tozzi@arcetri.astro.it \\ 3 Max-Planck-Institut für Astronomie, Königstuhl 17, 69117 Heidelberg, Germany \\ e-mail: hboehnha@mpia-hd.mpg.de \\ ${ }^{4}$ Isaac Newton Group of Telescopes, PO Box 321, 38700 Santa Cruz de La Palma, Tenerife, Spain \\ e-mail: pleisy@ing.iac.es
}

Received 20 August 2003 / Accepted 19 February 2004

\begin{abstract}
Comet Lee $(\mathrm{C} / 1999 \mathrm{H1})$ was observed on June 6, 1999 when it was at $r_{h}=0.98$ AU and $\Delta=1.195$ AU. The spectrophotometric observations, between 0.6 and $1 \mu \mathrm{m}$, were aimed at the detection of the CI $\left({ }^{1} \mathrm{D}\right)$ doublet $\lambda \lambda 9823 / 9850 \AA$. The non-detection of these lines, with a $3 \sigma$ flux upper limit of the order of $4.6 \times 10^{-17} \mathrm{erg} \mathrm{cm}^{-2} \mathrm{~s}^{-1}$, confirms the CO depletion already noted by other authors. Several $\mathrm{CN}$ and $\mathrm{NH}_{2}$ emission bands lie in that spectral range, making it possible to derive production rates for both species as $\sim 3.1 \times 10^{26} \mathrm{~s}^{-1}$ and $1.2 \times 10^{27} \mathrm{~s}^{-1}$, respectively. The oxygen forbidden line at $6300 \AA$ was used to obtain $Q_{\mathrm{H}_{2} \mathrm{O}}=(1.22 \pm 0.7) \times 10^{29} \mathrm{~s}^{-1}$. Assuming that $\mathrm{CN}$ and $\mathrm{NH}_{2}$ are directly produced by $\mathrm{HCN}$ and $\mathrm{NH}_{3}$, Comet Lee has a $\mathrm{HCN} / \mathrm{H}_{2} \mathrm{O} \approx 0.25 \%$ and $\mathrm{NH}_{3} / \mathrm{H}_{2} \mathrm{O} \approx 1 \%$ at $r_{h}=0.98 \mathrm{AU}$, in reasonable agreement with what has been found in other long-period comets. The structural analysis carried out on cometary images acquired with broad band $R$ Bessel filter clearly displays two pairs of ion rays likely produced by the $\mathrm{H}_{2} \mathrm{O}^{+}$doublet at 6198 and $6200 \AA$, wavelengths covered by the bandpass filter. Identical features are found in the images acquired with the Gunn $i$ filter.

The dust brightness profiles in the east-west direction do not deviate from a $\rho^{-m}$ law (with $0.7<m \leq 1.2$ ) as expected for a steady state model coma with a constant dust production rate and expanding at constant velocity. The dust production rate, as obtained from the $A f \rho$ parameter, is $\sim 500 \mathrm{~cm}$, which compared with the gas production rate classifies this comet as a dust poor one with relatively high (6.5-11.7) gas-to-dust mass ratio. Analysis of the normalized reflectivity gradient (i.e. continuum color) as a function of $\rho$ indicates a slight reddening of the solid component in the coma at large cometocentric distances, whereas the average dust color within an aperture of $20000 \mathrm{~km}$, centered at the nucleus, is $\sim 9 \%$ per $1000 \AA$. Mie scattering computations applied to an ensemble of particles indicate that the dust coma is characterised by a relative broad size distribution with a typical mean size of $1 \mu \mathrm{m}$. These grains might be composed of a mixture of silicates and icy material.
\end{abstract}

Key words. comets: individual: Comet C/1999 H1 (Lee) - comets: general

\section{Introduction}

Comet C/1999 H1 (Lee) was discovered on April 16, by Steven Lee from South Wales (IAUC 7144). Subsequent determination of the orbital elements by Marsden (MPEC 1999H06) classified this new object as a long-period ( $~ 81000 \mathrm{yr})$ comet in a "parabolic" orbit $(e=0.999742854)$ with semimajor axis at $2754 \mathrm{AU}$.

After discovery, as the comet would reach relatively short geocentric distance when approaching the perihelion, an extensive observation campaign took place. Weaver et al. (1999) carried out pre-perihelion infrared observations of Comet Lee detecting several bands of ethane, from which they estimated a production rate of $(0.7-1.4) \times 10^{27} \mathrm{~s}^{-1}$ on UT May 20.3,

Send offprint requests to: L. M. Lara, e-mail: lara@iaa.es

* Based on observations obtained at ESO La Silla within programme No. 60.A-9011(A). when the comet was at $r_{h}=1.22 \mathrm{AU}$ and $\Delta=0.86 \mathrm{AU}$. For the same date, the methane production rate was $\sim 4.5 \times 10^{26} \mathrm{~s}^{-1}$. Later on, during UT 20-29 May (1.10 $\leq r_{h} \leq 1.24 \mathrm{AU}$, $0.84 \leq \Delta \leq 1.02 \mathrm{AU})$, strong emissions were detected in the $\mathrm{C}-\mathrm{H}$ stretch region near $3.4 \mu \mathrm{m}$ and also broad emission feature centered near $3.27 \mu \mathrm{m}$ likely associated with PAHs. The methanol emission at $3.51 \mu \mathrm{m}$ made it possible to derive a production rate of the order of $2 \times 10^{27} \mathrm{~s}^{-1}$. During this observation campaign, no day-to-day temporal variability was observed at the $20 \%$ level.

Mumma et al. (2001) observed the comet in the near-IR $(1-5.5 \mu \mathrm{m})$ on June 2-3 and on August 19-21, 1999, before and after perihelion, searching for certain key organic species. Molecules such as water, carbon monoxide, methanol, methane, ethane, acetylene and hydrogen cyanide were detected and their production rates derived. The relative abundances found in this survey are similar to those found for 
comets Hyakutake and Hale-Bopp (two other comets that probably had their origin in the nebular region of the giant planets), excepting $\mathrm{CO}$, which is deficient in comet Lee by a factor of 5-10. This $\mathrm{CO}$ deficiency demonstrates that chemical diversity occurred within the formation region of the Oort-cloud comets.

Feldman et al. (1999) observed the comet pre-perihelion on June 7, 1999 making use of the Space Telescope Imaging Spectrograph (STIS) installed on the Hubble Space Telescope. The acquired long-slit spectra over the range $115-320 \mathrm{~nm}$ with a spatial resolution along the slit of $44 \mathrm{~km}$ projected at the comet showed strong emissions due to $\mathrm{OH}, \mathrm{CS}$ and continuum. The low dust/gas ratio implied by the relative brightness of $\mathrm{OH}$ to continuum also enabled the authors to detect $\mathrm{S}_{2}$ and $\mathrm{C}_{2}$. Therefore, Comet Lee is the third comet out of four where $\mathrm{S}_{2}$ has been positively identified (the other three are comet IRAS-Araki-Alcock C/1983 H1, Hyakutake C/1996 B2, and 153P/Ikeya-Zhang). The spatial profiles of CS and the continuum vary in time and are symmetric about the nucleus, while those of the $\mathrm{OH}$ bands display significant asymmetry.

Water was also observed with the Submillimeter Wave Astronomy Satellite, SWAS, (Melnick et al. 2000) by Nuefeld et al. (2000) on May 19-23, 1999. The production rate derived by these authors $\left(8 \times 10^{28} \mathrm{~s}^{-1}\right)$ is $50 \%$ lower than that derived by Biver et al. (2002) from contemporaneous radio observations of hydroxyl molecules. The data, taken over a period of 4.68 days, provide no evidence for variability. Post-perihelion observations, spanning from Sep. to Dec. 1999, with the same satellite (SWAS) (Chiu et al. 2001) indicate that the water production rate was steeply decreasing with heliocentric distance (as $r_{h}^{-5.5}$ in the range $r_{h}=1.3-1.7 \mathrm{AU}$ ) and was undetectable after Sep. 28.

In this paper, we present the morphological analysis of broadband images acquired in $R \# 642$ and $i \# 705$ filters, after bias and flat field correction as well as sky subtraction. The analysis of spectrophotometric observations of Comet Lee between 0.6 and $1.0 \mu \mathrm{m}$ taken on June 6, 1999 is also discussed. Two consecutive spectra were acquired aiming at the detection of the $\mathrm{CI}\left({ }^{1} \mathrm{D}\right)$ doublet at 9823.4 and $9849.5 \AA$. These spectra also contain information on gas emission from $\mathrm{OI}\left({ }^{1} \mathrm{D}\right), \mathrm{CN}$ and $\mathrm{NH}_{2}$, and continuum emission due to the scattering of the solar light by the cometary dust grains. Thus, we have obtained $\mathrm{CN}$ and $\mathrm{NH}_{2}$ production rates by using the customary Haser modeling (Haser 1957), $\mathrm{H}_{2} \mathrm{O}$ production rate by means of the $\mathrm{OI}\left({ }^{1} \mathrm{D}\right)$ prompt emission at $6300 \AA$, and $3 \sigma$ upper limit detection of the atomic carbon lines. Additionally, we have studied the continuum brightness profiles, the dust color as a function of projected cometocentric distance, $\rho$ and the dust production rate parameterized by $A f \rho$ (A'Hearn et al. 1984). Mie-scattering modeling has provided us with an approximate dust size distribution as well as quantitative composition.

\section{Observations and basic data reduction}

\subsection{Broadband imaging}

Comet C/1999 H1 (Lee) was observed at the European Southern Observatory, La Silla, Chile, preperihelion on June 6, 1999, when the comet was at 1.195, 0.979 AU geocentric and heliocentric distance, respectively. We used the EFOSC2 instrument mounted on the $3.6 \mathrm{~m}$ telescope $(2060 \times 2060$ pixels, including overscan pixels, pixel size: $0{ }^{\prime} 157$, usable FOV 5'.2× $5 ! 2)$. The telescope tracking was set to the proper motion of the comet and the sky conditions were photometric.

For telescope pointing and slit acquisition purposes, the comet was imaged with $R$ Bessel $\left(\lambda_{\mathrm{c}}=6431 \AA\right.$, $F W H M=1654 \AA)$ and Gunn $i\left(\lambda_{\mathrm{c}}=7931 \AA\right.$, $\left.F W H M=1256 \AA\right)$ filters. Additionally, sky flats were acquired with the same filters for bias and flatfield correction of the images. Since the main objective of the observation campaign was to obtain the long-slit spectra, no devoted standard stars were observed with these broadband filters. However, a summary of the EFOSC2 zero points is available at http://www.ls.eso.org/lasilla/sciops/efosc/docs/ Perf_ZeroPtSummary.html and we have used the $Z_{p}$ in $R$ and $i$ for June 6, 1999 to calibrate these broadband images in $A f$ (see Tozzi \& Licandro 2002). An approximate sky background level was determined from ring aperture measurements at a distance from the nucleus at which almost no coma light was found. A morphological analysis has also been carried out on the reduced images.

\subsection{Long-slit spectrophotometry}

Spectrophotometric observations were taken with a grating of 300 lines per mm, a slit width and length of $1^{\prime \prime}$ and $\sim 5^{\prime}$, respectively. This resulted in an observable spectral range between $6015 \AA$ and $10300 \AA$ with a wavelength scale of $2 \AA$ per pixel and a spatial scale of $2 \times 0$ '. 157 per pixel since the data were rebinned in the spatial direction. The slit of the spectrograph was orientated east-west, leading to spatial profiles that lie approximately parallel to the Sun-comet line projected on the plane of the sky (position angle of the Sun $(\mathrm{PA}) \approx 112^{\circ}$ ). For absolute calibration, we obtained observations of the spectrophotometric standard star LTT $7379\left(V=10^{\mathrm{m}} 23\right)$. The two acquired spectra were reduced using the ESO-MIDAS standard reduction context long for long-slit spectra. The spectra were bias subtracted, flatfielded, wavelength calibrated (using HeAr reference spectra), extinction corrected (using the standard extinction curve for La Silla) and finally flux calibrated. The cometary emission barely filled the slit and the sky flux could be determined form the edges of the frames. Details of the observations are given in Table 1.

\section{Data analysis and results}

\section{1. $2 D$ morphology}

Just for completeness, we have investigated the general spatial distribution of particles in the coma of Comet Lee from the acquired broadband images. The analysis of the existing asymmetries was carried out on the images after bias and flatfield correction, and sky subtraction. Either in $R$ Bessel or Gunn $i$, the comet shows the dust coma and an incipient dust tail, with a superimposed spherical coma from the gas emission at wavelengths covered by the transmission curve of the filters, as well 
Table 1. Details of the observations on June 6, 1999.

\begin{tabular}{|c|c|c|c|c|c|c|c|c|}
\hline $\begin{array}{l}\text { Time } \\
\text { UT }\end{array}$ & Band & $\begin{array}{c}\text { Exp. time } \\
\text { (s) }\end{array}$ & $\begin{array}{c}\Delta \\
\mathrm{AU}\end{array}$ & $\begin{array}{c}r_{h} \\
\mathrm{AU}\end{array}$ & $\begin{array}{c}\mathrm{km} \\
\text { per pixel }\end{array}$ & airmass & $\begin{array}{l}\text { PA } \\
\text { deg }\end{array}$ & $\begin{array}{c}\text { Phase } \\
\text { deg }\end{array}$ \\
\hline $22: 59: 33,23: 29: 59$ & Gunn $i$ & 20 & & & & $1.76-2.09$ & & \\
\hline $23: 07: 52-23: 51: 27$ & $R$ Bessel & 20 & 1.195 & 0.980 & 136 & $1.84-2.20$ & 111.5 & 54.59 \\
\hline $23: 40: 22-00: 06: 13$ & spectra & 600 & & & & $2.30-2.69$ & & \\
\hline
\end{tabular}

PA refers to the position angle of the extended Sun-target radius vector. Phase is the Sun-comet-observer angle. $r_{h}$ and $\Delta$ are the heliocentric and geocentric distances of the comet during our observations.

as some structures in the form of rays presumably produced by the $\mathrm{H}_{2} \mathrm{O}^{+}$doublet at $\sim 6200 \AA$.

To enhance the existing asymmetries and/or features, two different methods were applied: 1) adaptive Laplace filtering (Richter 1978, 1991; Boehnhardt \& Birkle 1994) and 2) radial renormalization (A'Hearn et al. 1986). The former method does not depend on the accuracy of the comet nucleus centering by a 2D Gaussian, and it is more sensitive to gradient changes on different scales depending on the width of the spatial filtering applied. The latter method uses the azimuthally averaged radial profile of the coma flux to create a 2 -dimensional image of the average coma flux level and divides it through the original image of the comet.

Figure 1 shows the coma of the comet before and after applying enhancement technique 1 to a median combined image in $R$ Bessel. The coma of Comet Lee shows up to four streams or jets (two of them clearly defined) in the anti-sunward direction. These streams are most likely produced by the line doublet at 6198 and $6200 \AA$ of the $(0,8,0)$ transition of the $\tilde{A}^{2} A_{1}-\tilde{X}^{2} B_{1}$ electronic system of the $\mathrm{H}_{2} \mathrm{O}^{+}$, as well as by the comparably strong $(0,6,0),(0,7,0)$ and $(0,9,0)$ bands. These streams have an approximate width of $5^{\circ}$ and the two most prominent ones are located at azimuthal angles of $287^{\circ}$ and $296^{\circ}$ as measured from north toward east.

Ion rays usually occur in pairs on either side of the ion tail, as they trace the magnetic field of the solar wind as it flows through the comet. The flow of the solar wind is decelerated by the addition of cometary ions to the wind. The deceleration is greatest near the nucleus, while on the outer edges of the coma, the solar wind continues to flow near its usual speed. This velocity shear gives the magnetic field lines, and the rays, their "hairpin" shapes.

It is known that significant changes in the ion rays can occurr; they first appear at fairly large angles to the ion tail, typically $45^{\circ}$, and then they brighten and converge towards the tail axis. The faster the solar wind speed at the comet, the more rapid the ion rays move. This process occurs over the course of typically $30 \mathrm{~min}-1 \mathrm{~h}$. To detect any change in the position angle of the ion rays, and since the comet was imaged during $\sim 45$ min, approximately, we have applied a Laplace filtering to the individual images. A slight convergence of the ion rays towards the tail can be seen in Fig. 2. The fact that these variations are not very large indicates that the solar wind speed at the comet might not have been very high at the time of our observations.
The adaptive Laplace filtering, applied to the Gunn $i$ images, enhances the ions rays, although their contrast is slightly worse, as expected from a fainter $\mathrm{H}_{2} \mathrm{O}^{+}$band at $\sim 7500 \AA$ within the filter bandpass.

The calibration in $A f$ was applied to median combined frames in $R$ Bessel and Gunn $i$. The parameter $A f \rho$ was introduced by A'Hearn et al. (1984); it represents a measurement unit related to the dust production rate of a comet. Making use of the available $Z_{p}$, we have computed $A f \rho_{R} \sim 700 \mathrm{~cm}$ and Af $\rho_{i} \sim 590 \mathrm{~cm}$ at $\rho \approx 40000 \mathrm{~km}$. Note that the bandpass of these filters certainly covers several emissions due to $\mathrm{C}_{2}$, $\mathrm{NH}_{2}, \mathrm{H}_{2} \mathrm{O}^{+}$and $\mathrm{CN}$, besides the solar radiation scattered by the dust, and thus the obtained $A f \rho$ parameters are upper limits to the more realistic ones obtained from the spectroscopic measurements where relatively clean continuum regions can be selected.

\subsection{Gas and dust spatial profiles}

The gas $\left(\mathrm{NH}_{2}, \mathrm{CN}\right.$, OI and $\left.\mathrm{CI}\right)$ and dust coma have been studied from the two acquired long-slit spectra in the east-west direction. After flux calibration, both spectra were recentered at the pixel which presumably contains the cometary nucleus and aligned along the slit to the optocenter. The resulting spectrum was then considered to study the $\mathrm{CN} A^{2} \Pi-X^{2} \Sigma^{+}$red system, emission bands of $\mathrm{NH}_{2} \tilde{A}\left(0, v_{2}^{\prime}, 0\right)-\tilde{X}(0,0,0)$ (hereafter called the " $0, v_{2}^{\prime}, 0$ band"), atomic oxygen and atomic carbon prompt emissions at 6300/6364 and 9823/9850 A respectively, as well as the continuum profiles in the east-west direction, dust production rate (as measured from the $A f \rho$ parameter), and approximate size distribution and composition of the cometary dust grains.

Several emission features are visible in both cometary spectra (see Fig. 3, upper panel): the $\mathrm{OI}\left({ }^{1} \mathrm{D}\right)$ cometary feature at $6300 \AA$, the $\mathrm{NH}_{2}$ bands for $v_{2}^{\prime}=9$ (partially), 8, 7, 6 and 5 and the $\mathrm{CN}$ red system bands $2-0,3-1,1-0$ and $2-1$. Superimposed on the proper cometary emissions, important telluric bands such as the $\mathrm{O}_{2} \mathrm{~B}$ and $\mathrm{A}$, several $\mathrm{H}_{2} \mathrm{O}$ and $\mathrm{OH}$ bands (longward of $9000 \AA$ ) can be seen, as well as the atmospheric oxygen forbidden line at $6300 \AA$.

To obtain the pure gas emission features, a onedimensional synthetic dust spectrum was obtained using a solar high resolution spectrum (Kurucz et al. 1984) transformed (or calibrated) in flux according to the solar spectrum distribution measured by Labs et al. (1987). This spectrum was convolved with the 


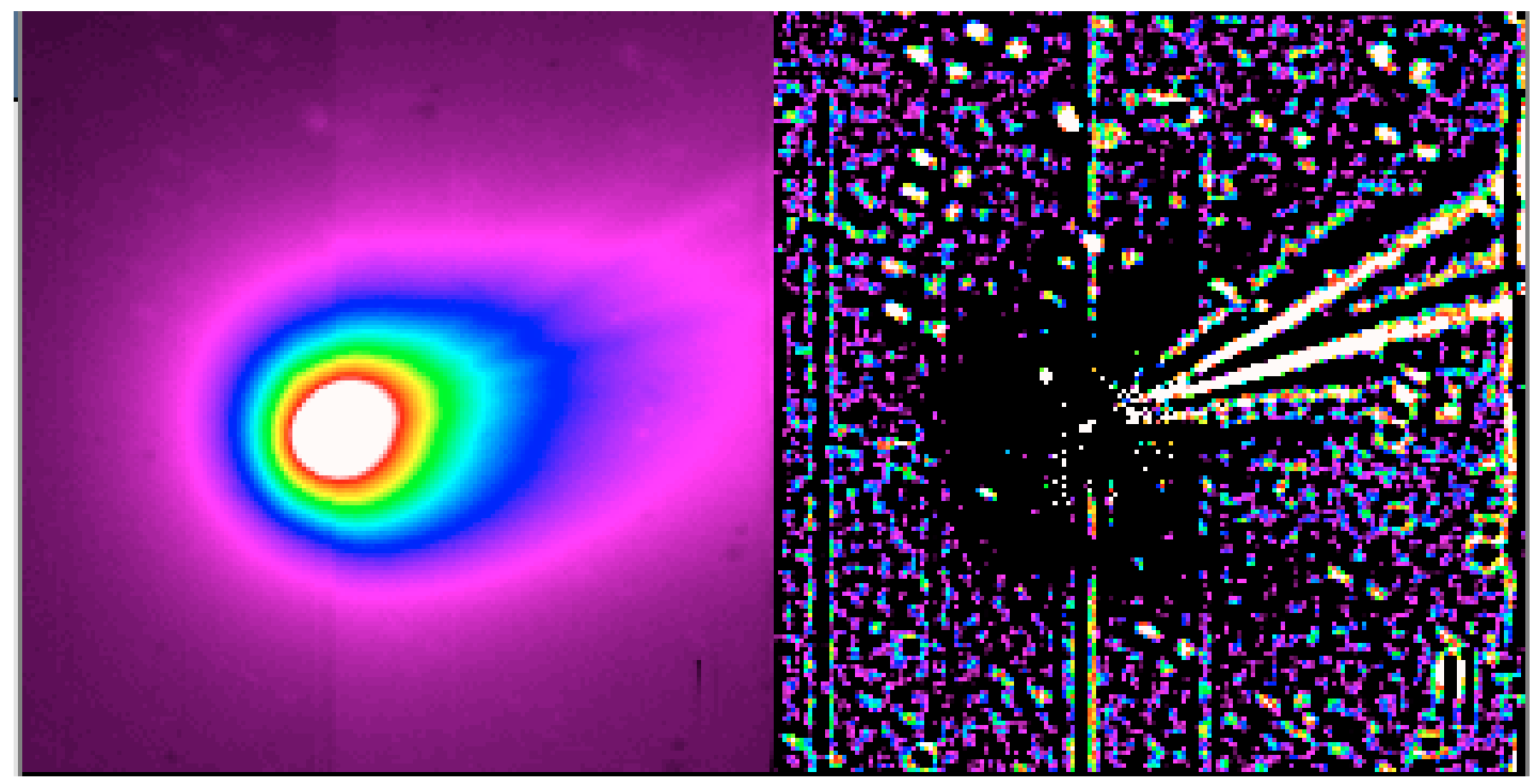

Fig. 1. Comet Lee (C/1999 H1) as imaged in the $R$ Bessel filter (left) and processed image (Laplace filter with a filter width of 31 pixels) to enhance structures. The angular scale for both images is $4.58 \times 5.25$, which is $200000 \times 230000 \mathrm{~km}$ at the comet distance. North is up and East to the left. Note that up to two clear streams, that are due to $\mathrm{H}_{2} \mathrm{O}^{+}$, are enhanced by the Laplace filtering. The position angle of these structures is $287^{\circ}$ and $296^{\circ}$ as measured from north toward east. Each of these features has a width of $\sim 5^{\circ}$. The vertical lines are artifacts from bad columns, whereas the dotty features are background stars.

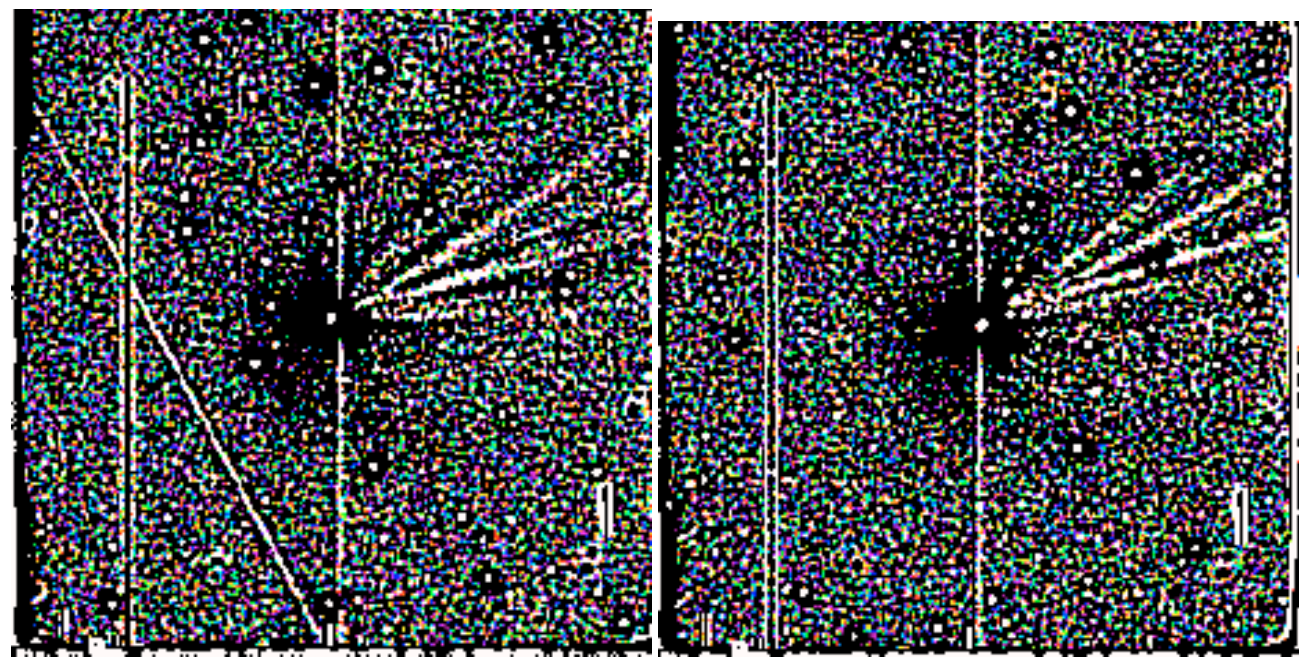

Fig. 2. The ion rays of Comet C/1999 H1 (Lee): images acquired at 23:07:52 UT (left) and 23:51:27 UT (right) after applying an adaptive Laplace filter (width of 17 pixels). Viewing geometry is as in Fig. 1, whereas the the field of view is 323 .' $42 \times 323$ ". 42 for both frames, which is $280300 \times 280300 \mathrm{~km}$ at the comet distance. Comet nucleus is at the center of the frame. A slight coarsening of the ion rays towards the tail axis can be seen.

instrumental point spread function and adjusted to account for the intrinsic dust color of the comet. The latter is deduced from the slope of the cometary spectrum measured in wavelength regions without known gas contamination. From this, a two dimensional synthetic cometary dust spectrum was created using the cometary dust profiles along the slit, extracted from wavelength regions without gas contamination. Finally, the difference between the cometary and the synthetic dust spectrum gives the cometary gas spectrum. The result can be checked in Fig. 3 where the original cometary spectrum (i.e. the observed one before sky subtraction) and the cometary gas spectrum, both integrated in the spatial direction along the slit, are shown. Note that the cometary gas spectrum, after sky subtraction too, contains practically no telluric lines and/or bands and only the pure cometary gas emissions is visible, that is, the continuum level is about zero. This procedure was applied to each spectrum. After checking the similarity in the intensity of every gas band and/or line in the two spectra, they were median 

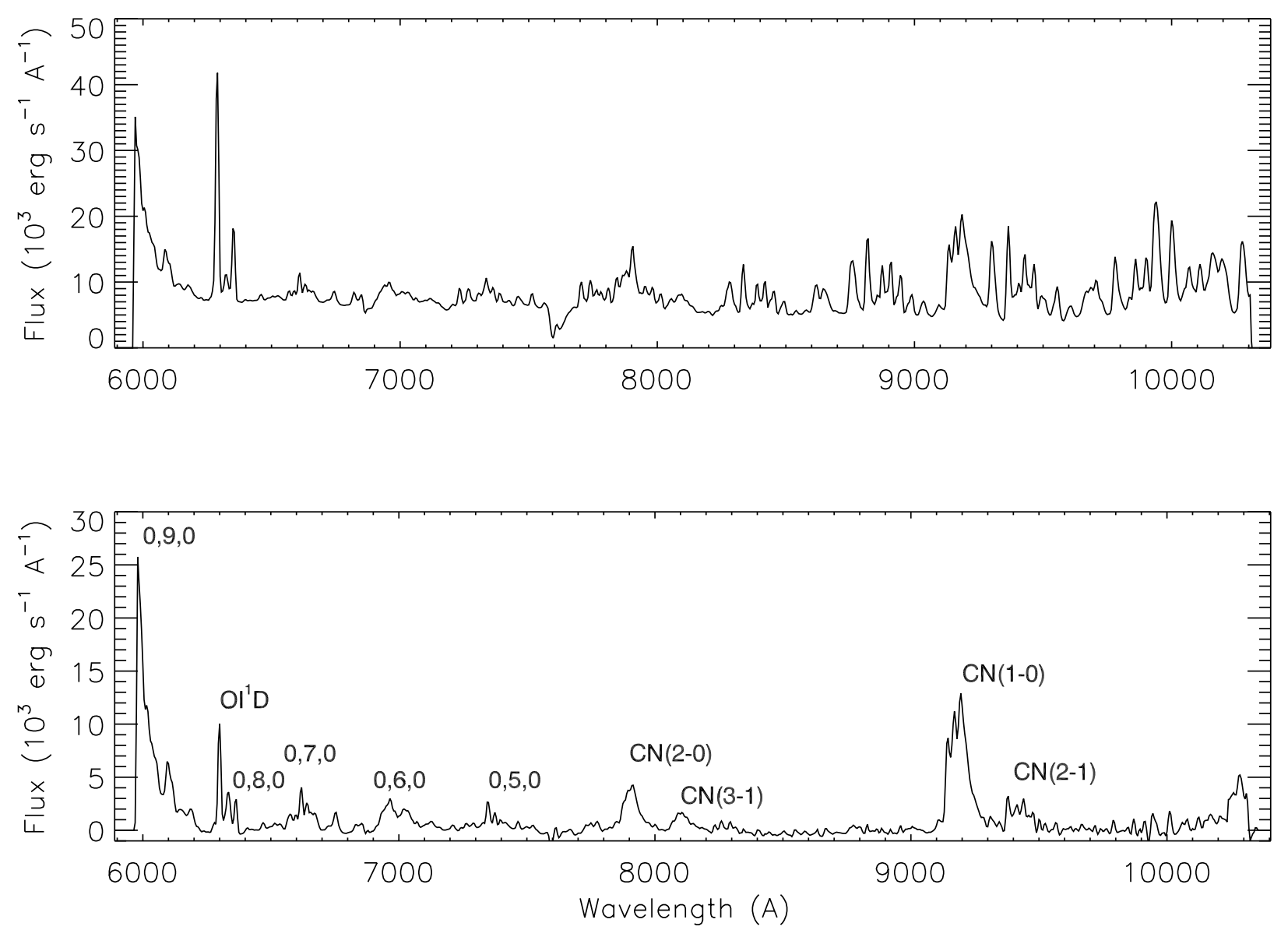

Fig. 3. Observed and gas spectrum of Comet Lee (C/1999 H1) spatially integrated along the slit. In the observed cometary spectrum, the sky values have not been subtracted so that it clearly shows the oxygen telluric line, the $\mathrm{O}_{2} \mathrm{~B}$ and A bands at $\sim 6800$ and $\sim 7600 \AA, \mathrm{H}_{2} \mathrm{O}$ at $\sim 8200 \AA$, and $\mathrm{OH}$ band longward $9000 \AA$. In the lower panel, pure cometary emissions is seen: the $\mathrm{CN}$ red system as well as the $\left(0, v_{2}^{\prime}, 0\right) \mathrm{NH}_{2}$ bands (with the blended OI lines at $6300 \AA$ and at $6364 \AA$ ).

combined to increase the $\mathrm{S} / \mathrm{N}$. This final spectrum is the one considered for the dust and gas analysis presented in the following sections.

\subsection{1. $\mathrm{OI}+\mathrm{NH}_{2}$ and $\mathrm{CN}$}

It is widely known that the $(0,8,0) \mathrm{NH}_{2}$ band is blended with the $\mathrm{OI}\left({ }^{1} \mathrm{D}\right)$ lines, that the $(0,7,0)$ is most likely contaminated by the $C_{2} \Delta v=-3$ sequence, and that the $(0,6,0)$ and $(0,5,0)$ bands are relatively spread and mixed with strong telluric absorptions. The case of the $(0,8,0) \mathrm{NH}_{2}$ band blended with the oxygen forbidden lines at 6300 and $6364 \AA$ has been previously studied by Fink (1994), Hicks \& Fink (1997), and Fink et al. (1999). To properly obtain the $\mathrm{NH}_{2}$ and $\mathrm{OI}\left({ }^{1} \mathrm{D}\right)$ uncontaminated flux, both species need to be corrected. High resolution spectra of several comets, including P/Halley (Arpigny et al. 1987a; Combi \& McCrosky 1991) show that most of the $(0,8,0) \mathrm{NH}_{2}$ emission consists of a peak centered near $6290 \AA$, and thus blended with the $6300 \AA \mathrm{OI}\left({ }^{1} \mathrm{D}\right)$ line, and a pure $\mathrm{NH}_{2}$ feature centered near $6335 \AA$, as well as a number of weaker features. The average ratio $\frac{F_{6290}}{F_{6335}}$ for eight comets is about 0.87 , while five observations of comet $\mathrm{P} /$ Halley at varying heliocentric distance yielded 0.86 . This factor was determined by Arpigny et al. (1987b) and it seems to be quite robust over a wide range of geocentric and heliocentric distances, as well as being independent of spectral resolution as long as the OI and the $\mathrm{NH}_{2}$ peaks can be reasonably well resolved. To obtain the pure $6300 \AA$ OI emission, we thus multiplied the $6335 \AA \mathrm{NH}_{2}$ peak by this number, and subtracted this amount of $\mathrm{NH}_{2}$ from the $\mathrm{OI}+\mathrm{NH}_{2}$ blend at $6300 \AA$. Next, to obtain the total $\mathrm{NH}_{2}$ flux of the $(0,8,0)$ band, the flux measured at $6335 \AA$ was multiplied by 2 since the emission at that wavelength comprises about 0.49 of the total $\mathrm{NH}_{2}$ flux (see Fink 1994).

Besides the difficulty in extracting pure $\mathrm{NH}_{2}$ emission from some of the bands appearing in the cometary spectrum (see Fig. 3), the exact resonance fluorescence $g$-factors are not known. The $g$-factors for the $\mathrm{NH}_{2}$ bands commonly used are those in Tegler \& Wyckoff (1989) until 1994, and the same $g$-factors halved later on. This change was required because Arpigny (1995) pointed out that the even bands $v_{2}^{\prime}=8$, 6, etc. and the odd bands $v_{2}^{\prime}=9,7$, etc., each account for half the population of $\mathrm{NH}_{2}$ molecules in the ground state. Recently, the $\mathrm{NH}_{2}$ fluorescence efficiencies have been re-calculated by Kawakita \& Watanabe (2002). These authors computed the $g$ factors as a function of heliocentric distance, finding that the fluorescence efficiencies for even $-v_{2}^{\prime}$ bands are approximately proportional to $r_{h}^{-n}$, where $n=1.5-1.6$, while those for the odd $-v_{2}^{\prime}$ bands cannot be approximated by the $r_{h}^{-n}$ function. 
Table 2. CN emission bands extracted from the spectra, their corresponding $g$-factors, and derived production rates.

\begin{tabular}{cccc}
\hline \hline Species & $\begin{array}{c}\text { Spectral region } \\
(\AA)\end{array}$ & $\begin{array}{c}g \text {-factor } \\
\left(\mathrm{erg} \mathrm{s}^{-1} \mathrm{~mol}^{-1}\right)\end{array}$ & $\begin{array}{c}Q \\
\left(10^{26} \mathrm{~s}^{-1}\right)\end{array}$ \\
\hline $\mathrm{CN}(2-0)$ & $7822-8039$ & $2.70 \times 10^{-14}$ & $2.97 \pm 0.13$ \\
$\mathrm{CN}(3-1)$ & $8032-8213$ & $9.85 \times 10^{-15}$ & $3.77 \pm 0.23$ \\
$\mathrm{CN}(1-0)$ & $9109-9323$ & $8.91 \times 10^{-14}$ & $3.24 \pm 0.17$ \\
$\mathrm{CN}(2-1)$ & $9342-9528$ & $2.33 \times 10^{-14}$ & $2.55 \pm 0.12$ \\
\hline \multicolumn{5}{c}{} & & $3.13 \pm 0.51^{a}$ \\
\hline
\end{tabular}

${ }^{a}$ Mean CN production rate on June 6, 1999.

The $\mathrm{C}_{2}$ contamination of the $v_{2}^{\prime}=7,6 \mathrm{NH}_{2}$ bands becomes clear when comparing the east-west profiles derived from the $(0,8,0)$ band with the $(0,7,0)$ and $(0,6,0)$ bands: the one deduced from the $(0,8,0)$ is noticeably steeper (and more characteristic of arising from $\mathrm{NH}_{3}$ photodissociation) than the ones resulting either from the $(0,6,0)$ or the $(0,7,0)$ band which are much flatter, reflecting the contamination by the $\Delta v_{2}=-3 \mathrm{C}_{2}$ emissions. Therefore, only the $\mathrm{NH}_{2}$ profile derived from the $(0,8,0)$ band will be used for the gas analysis of the coma of Comet Lee. The corresponding $g$-factor is $3.63 \times 10^{-15} \mathrm{erg} \mathrm{s}^{-1} \mathrm{~mol}^{-1}$ according to Tegler \& Wyckoff (1994), or $7.79 \times 10^{-15} \mathrm{erg} \mathrm{s}^{-1} \mathrm{~mol}^{-1}$ as inferred from the data in Table 1 of Kawakita \& Watanabe (2002) interpolated at $r_{h}=0.98 \mathrm{AU}$ (heliocentric distance of Comet Lee during our observations).

The $\mathrm{CN}$ emission bands in the red system are strongly peaked, with well-defined boundaries and good contrast with respect to the continuum. Thus, their extraction from the cometary gas spectrum is straightforward according to the ranges listed in Table 2. The column densities for each band are derived making use of the $g$-factors adopted from Fink (1994) and also listed in Table 2.

We have tried to fit the $\mathrm{CN}$ and $\mathrm{NH}_{2}$ column density profiles derived from each band in the east-west direction under the assumption of constant production rates, i.e. a steady state model, by applying the Haser modeling (Haser 1957) with parent expansion velocities given by the expression $v_{\mathrm{p}}=$ $0.86 \times r_{h}^{-0.4} \mathrm{~km} \mathrm{~s}^{-1}$ (Biver et al. 2000), while $v_{d}$ is held constant and equal to $1 \mathrm{~km} \mathrm{~s}^{-1}$ for both $\mathrm{CN}$ and $\mathrm{NH}_{2}$. The Haser equivalent (see Combi \& Delsemme 1980) scalelengths $\left(l_{p}, l_{d}\right)$ best fitting the column density profiles are $(48500,255000) \mathrm{km}$ for every $\mathrm{CN}$ profile. The obtained $\mathrm{CN}$ production rates are listed in Table 2. In the case of $\mathrm{NH}_{2}$, a theoretical profile obtained with $l_{p}=7500 \mathrm{~km}, l_{d}=70000 \mathrm{~km}$, and production rate $(5.52 \pm 0.03) \times 10^{27} \mathrm{~s}^{-1}$ (if the $g$-factor is from Tegler \& Wyckoff 1994) or $(1.18 \pm 0.01) \times 10^{27} \mathrm{~s}^{-1}$ (if the fluorescence factor is from Kawakita \& Watanabe 2002) best resembles the observations. Figure 4 shows the observed profiles in the eastwest direction together with the theoretical profiles best reproducing the observed ones.

\subsection{2. $\mathrm{H}_{2} \mathrm{O}$}

The water production rate is derived from the flux measurement of the forbidden $\mathrm{O}^{1} \mathrm{D}$ line at $6300 \AA$. This oxygen line, and the one at $6364 \AA$, are not produced by resonance fluorescence, but by the prompt emission produced by the photodissociation of $\mathrm{H}_{2} \mathrm{O}$, which directly populates the $\mathrm{OI}\left({ }^{1} \mathrm{D}\right)$ and $\mathrm{OI}\left({ }^{3} \mathrm{P}\right)$ states. The reported branching ratio to that channel $(8.2 \%$ by Festou \& Feldman 1981 and $8.3 \%$ by Budzien et al.1994) probably has an uncertainty of 20-30\%. Due to its short lifetime $(\sim 110 \mathrm{~s})$, the $\mathrm{O}^{1} \mathrm{D}$ flux profile faithfully traces out the $\mathrm{H}_{2} \mathrm{O}$ parent molecule distribution, and its emission is comparatively narrow. From the total luminosity $L$ (in photons $\mathrm{s}^{-1} \mathrm{~m}^{-2}$ ) at $6300 \AA$, and integrating the spectrum of Comet Lee along the full spatial slit length (i.e. $\approx 1 \times 10^{5} \mathrm{~km}$ ), we have derived a $\mathrm{H}_{2} \mathrm{O}$ production rate of $(1.22 \pm 0.7) \times 10^{29} \mathrm{~s}^{-1}$ for a branching ratio of $8.3 \%$ in the process $\mathrm{H}_{2} \mathrm{O}+\mathrm{h} v \rightarrow \mathrm{O}\left({ }^{1} \mathrm{D}\right)+\mathrm{H}_{2}$ at $\lambda<1360 \AA$.

\subsection{3. $\mathrm{Cl}$}

The abundance of carbon atoms in the metastable ${ }^{1} \mathrm{D}$ state near the cometary nucleus provides information on one of the main sources of carbon in the cometary coma. The prompt emission of the ${ }^{1} \mathrm{D}-{ }^{3} \mathrm{P}$ doublet takes place at $9823 / 9850 \AA$. The ${ }^{1} \mathrm{D}$ state of carbon is metastable, with a lifetime of 4077 s (Hibbert et al. 1993), likely produced by CO photodissociation (Tozzi et al. 1998). At the available spectral resolution $(F W H M=2 \AA)$, we have searched for the $\mathrm{C}\left({ }^{1} \mathrm{D}\right)$ doublet at 9823 and $9850 \AA$. No clear spectral feature was found in the inner coma $(\rho \leq$ $12250 \mathrm{~km}$, i.e. one "scale length" assuming $\tau \approx 4077 \mathrm{~s}$ and $v_{d} \approx 3.9 \mathrm{~km}$, Huebner et al. 1992) at any of these wavelengths. A $3 \sigma$ flux upper limit for the doublet $9823 / 9850 \AA$ has been computed to be $(4.56 \pm 0.52) \times 10^{-17} \mathrm{erg} \mathrm{cm}^{-2} \mathrm{~s}^{-1}$.

\subsubsection{Dust brightness profiles}

The two dimensional cometary dust spectrum has allowed us to study the behavior of the dust and of its color as a function of $\rho$. Three continuum regions at $6250 \AA, 7230 \AA$ and $8900 \AA$ can be selected for the study of the cometary dust. Figure 5 shows the brightness profiles derived from the spectrum for the continua centered at $6250 \AA$ and $8900 \AA$. All of these profiles, either in east or west direction, can be adequately fit by the law $\log B \sim-m \log \rho$ with $m \sim 1$ (see Table 3). This is consistent with a steady state coma for a constant dust production rate and long-lived grains expanding radially outward, where the spatial number density should decrease as $r^{-2}$, where $r$ is the nucleocentric distance. Consequently, the projected surface brightness would decrease as $\rho^{-m}$ with $m=1$.

\subsubsection{A f $\rho$}

Estimates of the dust production in comets are usually made by means of the parameter $A(\theta) f \rho$ (A'Hearn et al. 1984). The expression to compute the equivalent radius $\rho$ of a rectangular aperture (as used in our observations) is taken from Roettger (1991), yielding 2'. 35 (i.e. $2040 \mathrm{~km}$ at the comet distance). 


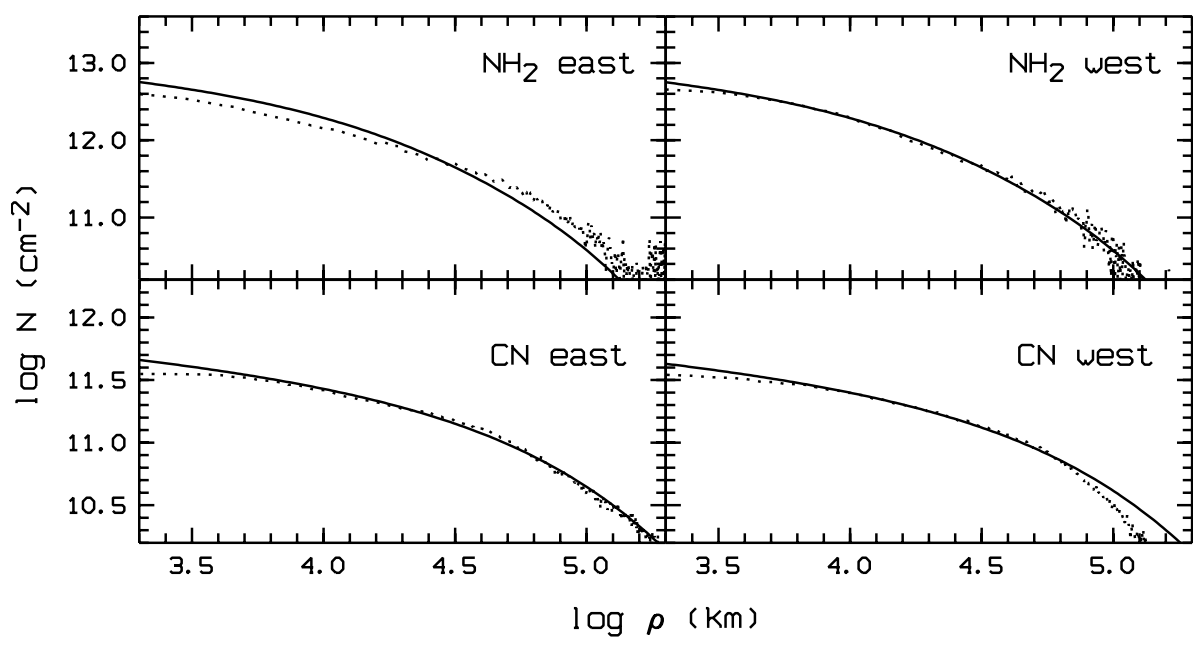

Fig. 4. $\mathrm{CN}$ and $\mathrm{NH}_{2}$ column density profiles of Comet Lee (dots) in the east-west direction. The solid lines represent the results obtained by means of the Haser model with equivalent scalelengths and production rates listed in Table 2 for $\mathrm{CN}(1-0)$ and as mentioned in the text for $\mathrm{NH}_{2}$.

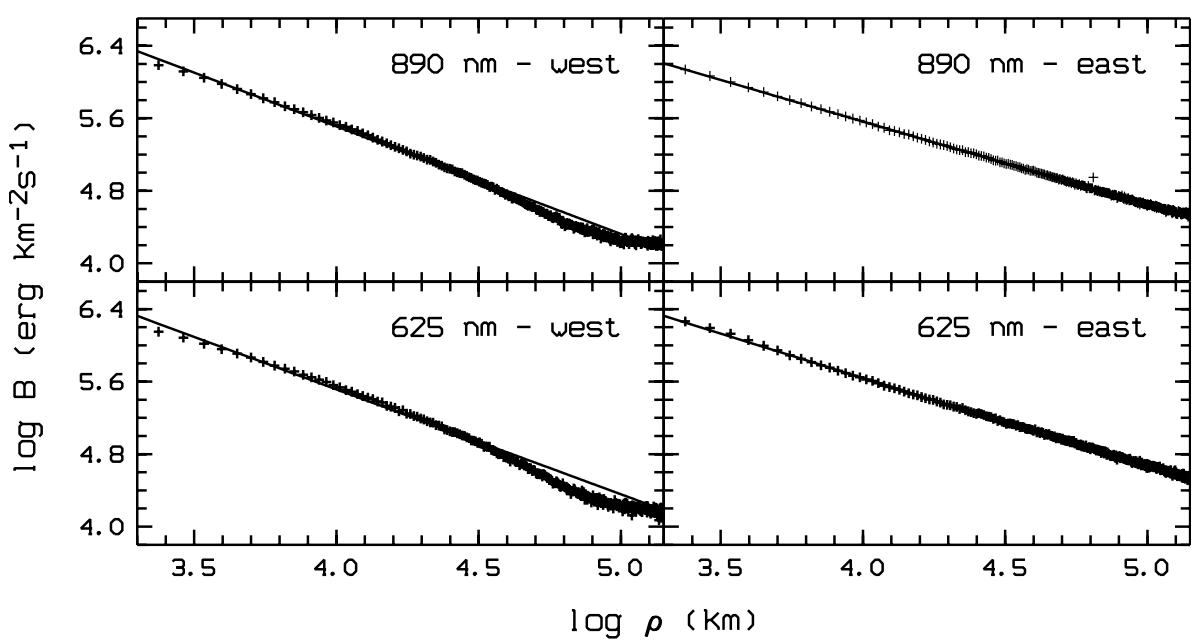

Fig. 5. Brightness profiles of Comet Lee in two different continuum regions. The profiles were obtained from the median averaged spectrum in the east-west direction, representing approximately the sun-tail direction. The surface brightness, $B$, is plotted against the projected radius, $\rho$ in double logarithmic representation. Dots represent the observed profiles and the solid lines are the best fits verifying $\frac{\mathrm{d}(\log B)}{\mathrm{d}(\log \rho)}=-m$ starting at $\log \rho \geq 3$.2, with slopes listed in Table 3 .

Table 3. Slope of linear fits in Fig. 5.

\begin{tabular}{ccc}
\hline \hline $\begin{array}{c}\text { Spectral range } \\
(\AA)\end{array}$ & West & Slope $^{a}$ \\
\hline $6231-6267(6250)$ & $1.16 \pm 0.03$ & $0.978 \pm 0.010$ \\
$6800-6900(6850)$ & $1.20 \pm 0.03$ & $0.966 \pm 0.006$ \\
$7188-7266(7230)$ & $1.19 \pm 0.03$ & $0.917 \pm 0.006$ \\
$8866-8942(8900)$ & $1.00 \pm 0.02$ & $0.718 \pm 0.008$ \\
\hline
\end{tabular}

${ }^{a}$ Linear fit for the region $3.2 \leq \log \rho \leq 4.6$ (i.e. $1600 \leq \rho \leq$ $40000 \mathrm{~km})$.

From the flux in the continuum (6800-6900 $\AA$ ), we have calculated $A(\theta) f \rho=496 \mathrm{~cm}$, below the upper limit computed from the broadband images in $R$ Bessel and Gunn $i$, as expected.
Following A'Hearn et al. (1995), the gas-to-dust mass ratio can be evaluated by

$\log \left(\frac{M_{\text {gas }}}{M_{\text {dust }}}\right)=\log [Q(\mathrm{OH}) / A f \rho]-25.4$.

According to this expression, and considering the simultaneous $Q_{\mathrm{OH}}$ (direct observations by Feldman et al. 1999 at $3085 \AA$ and by Biver et al. 2000 at $18 \mathrm{~cm}$ or derived from water production rates as $0.90 \times Q_{\mathrm{H}_{2} \mathrm{O}}$, this work and Neufeld et al. 2000) and the $A f \rho$ parameter here presented $(\sim 500 \mathrm{~cm})$, the gas-to-dust mass ratio is bracketed between 6.5 and 11.7 at $r_{h}=0.98 \mathrm{AU}$.

\subsubsection{Dust color}

As the long-slit spectrum covers a wide wavelength range, and contains spatial information in approximately Sun-tail direction, the color of the cometary dust vs. the projected cometocentric distance $\rho$ can be easily derived. The bandpass representing continuum emissions are listed in Table 3. After 


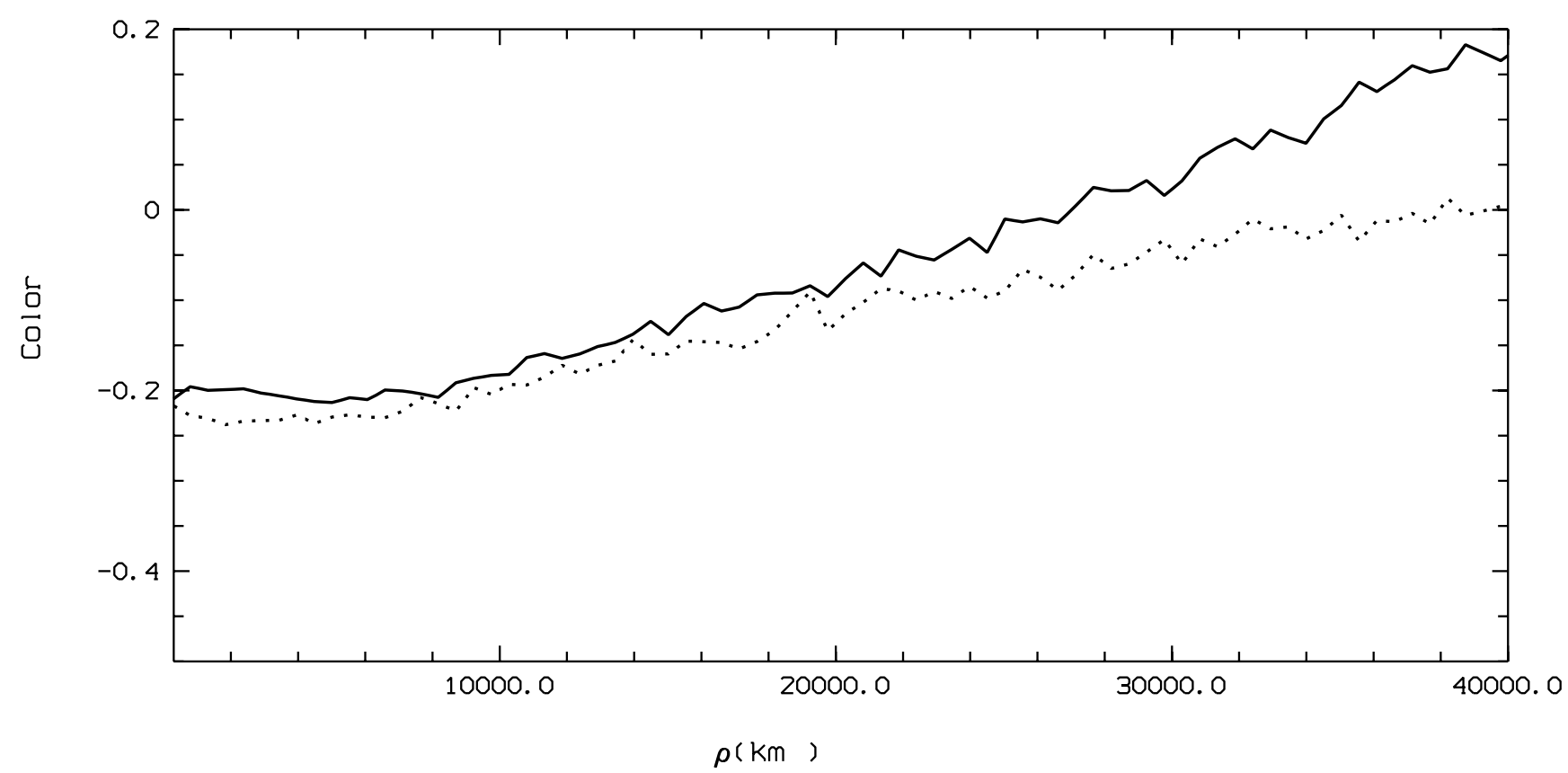

Fig. 6. Color of the dust in the coma of C/1999 H1 (Lee) in the west (solid line) and east (dotted line) direction as computed from Eq. (1) for $8400 \leq \lambda_{L} \leq 8500 \AA$ and $6800 \leq \lambda_{S} \leq 6900 \AA$. Note the increasing reddening of the dust at $\rho \geq 10000 \mathrm{~km}$ in both directions. At larger cometocentric distances, the differences in east-west direction cannot be attributed to significant variations in the physical and/or chemical properties of the cometary dust since at $\rho>30000 \mathrm{~km}$ the error due to the contribution of the sky is getting larger.

re-centering the spectrum at each wavelength range at the optocenter as determined by fitting a one-dimensional Gaussian to the inner part of the coma, the intensity peaks are all located within a fraction $(\sim 0.2)$ of a pixel, and the color of the dust is computed as

$$
C_{S-L}=\ln \left(\frac{F_{L}}{F_{S}}\right)
$$

where $F_{S}, F_{L}$ are the averaged reflected flux of the cometary dust, as a function of $\rho$, in the shorter and the longer wavelength ranges, respectively (see Kolokolova et al. 2001).

Regardless of what the continuum bandpass we consider, the color of the dust is rather constant at every $\rho$ in the east and west direction as shown in Fig. 6. In both directions, there seems to be a slight reddening of the cometary dust at increasing $\rho$ from the nucleus. By averaging the cometary emission in two spectral regions free of gas contamination (i.e. $\lambda_{L}=(8450 \pm 50) \AA$ and $\left.\lambda_{S}=(6850 \pm 50) \AA\right)$, we have also computed an intrinsic reddening of the dust of $\sim 9 \%$ per $1000 \AA$.

\subsubsection{Size distribution and composition of the dust grains}

Given this steady behavior of the dust color and of the canonical $\log B \approx-1 \log \rho$ indicating that the dust grains are longlived while traveling outward, we have tried to obtain some approximate information on the size distribution and likely composition of the dust grains by applying simple Miescattering theory (Bohren \& Huffman 1983) to a log-normal size distribution (see Hansen \& Travis 1974) of homogeneous spherical particles:

$n(a)=\frac{1}{(2 \pi)^{1 / 2} \sigma_{m}} \frac{1}{a} \exp \left[-\left(\ln a-\ln a_{m}\right)^{2} /\left(2 \sigma_{m}^{2}\right)\right]$.

By varying the mean radius $a_{m}$, the width of the distribution $\sigma_{m}$ and the optical constants (i.e. the complex refractive index $m=n-i k$ ), we have found that the best agreement (see Fig. 7) between observations and computations is reached for an ensemble of particles whose mean radius is $1 \mu \mathrm{m}, \sigma_{m}=1.5$, with a complex refractive index representative of a mixture of silicates (i.e. modified astronomical silicate) and icy grains $(m=1.33-0 i)$.

\section{Discussion}

The analysis of CCD images and of long-slit spectra of Comet Lee in the red branch of the spectrum has provided morphological and compositional information on the coma of another long-period comet recently visiting the inner Solar System.

The morphological analysis has been carried out on broadband images. Regarding the dust, besides the spherical coma no other dust features have been found. However, the existence of ion rays becomes quite clear after applying enhancement techniques. These ion rays are mainly produced by the line doublet at 6198 and $6200 \AA$ of the $(0,8,0)$ transition of the $\tilde{A}^{2} A_{1}-\tilde{X}^{2} B_{1}$ electron system of $\mathrm{H}_{2} \mathrm{O}^{+}$. Being usually produced in pairs on either side of the tail at a quite large angle of $\sim 45^{\circ}$ or more, they converge to the tail axis in a relatively short time ( $\sim 30-60 \mathrm{~min})$ From the first to the last comet image in $R$ Bessel on June 6, 1999, obtained 45 min apart, there 


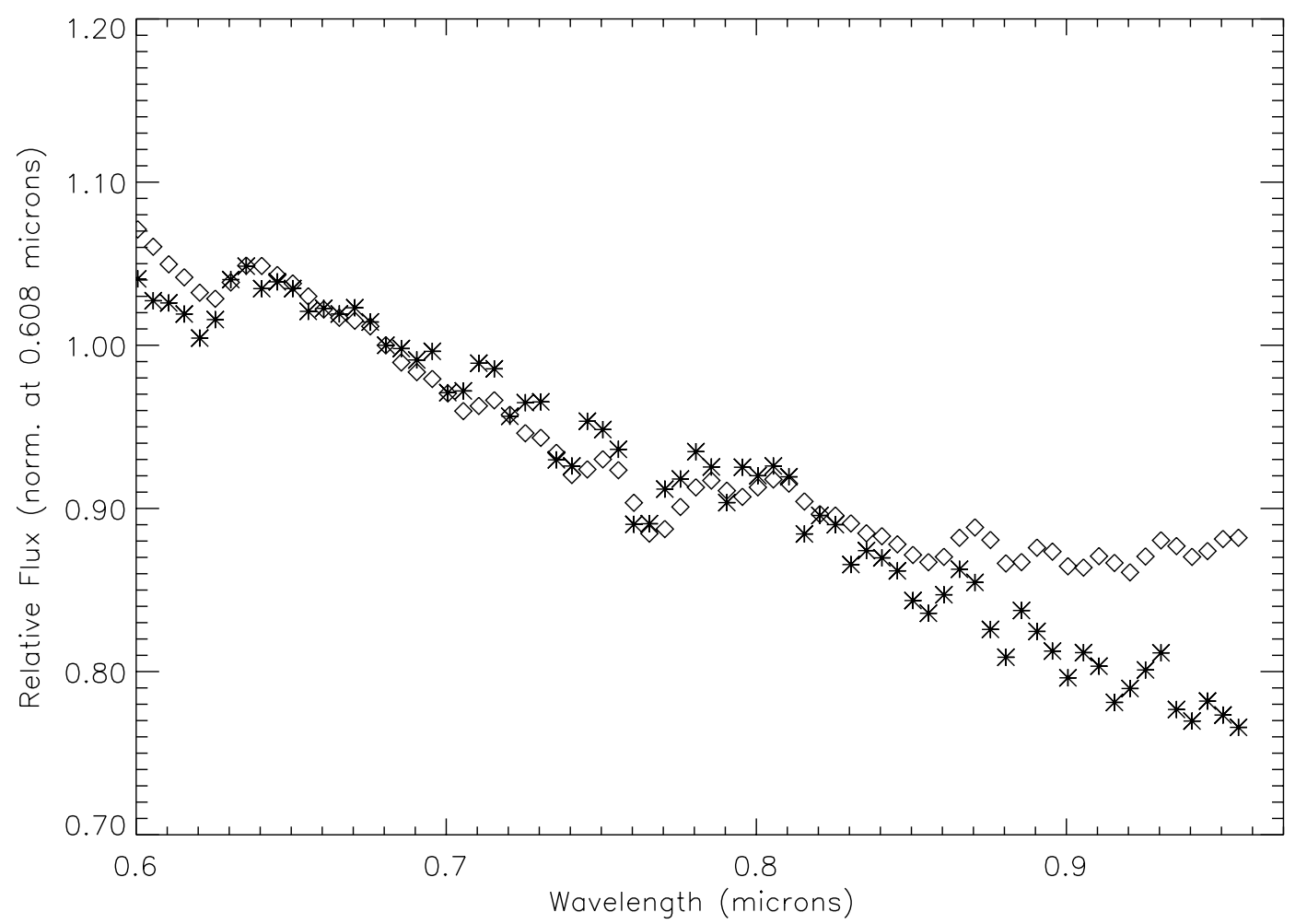

Fig. 7. Comparison between the observed light scattered by the cometary dust in C/1999 H1 (Lee) and that computed by means of the Mie-scattering theory. Data and theoretical results are normalized at $6805 \AA$. Asterisks refer to observations and diamonds to Mie-theory computations.

was a variation in the position angle of the two most prominent ion rays in $\sim 3^{\circ}$. The lack of images acquired with interference filters $\left(\mathrm{CN}, \mathrm{C}_{2}, \mathrm{C}_{3}, \mathrm{OH}\right.$, etc.) does not allow us to ascertain whether other gas structures are superimposed to the spherical coma. Furthermore, neither the $\mathrm{CN}$ nor the $\mathrm{NH}_{2}$ column density, as derived from the east-west profiles in every band, show any noticeable asymmetry in that particular direction. There is a slight overabundance of $\mathrm{NH}_{2}$ to the west at cometocentric distances lower than $\sim 20000 \mathrm{~km}$.

The CN band complex gives relatively consistent results, as seen in Table 2. The production rate derived from the $\mathrm{CN}(3-1)$ band is higher than that derived from the other bands, whereas that deduced from the (2-1) band is much lower. As mentioned by Fink (1994), the CN system is mixed with a number of highly variable $\mathrm{H}_{2} \mathrm{O}$ bands. More concisely, the $\mathrm{CN}(2-0)$ band lies just to the redward continuum region of a strong telluric water band. Furthermore, the published fluorescence efficiencies or $g$-factors might be affected by an overall uncertainty of $\leq 20 \%$ (Fink 1994). The average $\mathrm{CN}$ production rate is $(3.13 \pm 0.51) \times 10^{26} \mathrm{~s}^{-1}$, whereas discarding the highest derived $\mathrm{CN}$ production rate gives an average value of $(2.92 \pm$ $0.34) \times 10^{26} \mathrm{~s}^{-1}$. In both cases, the error is approximately $\leq 16 \%$, that is, $Q_{\mathrm{CN}}$ can be considered as realistic within the uncertainties derived from the inaccuracy of the $g$-factors and the calibration errors (note that the airmass of the comet spectrum was very high and that the flux calibration might have suffered from this). The required $\mathrm{CN}$ parent Haser equivalent scalelength to fit the observational data (i.e. $48500 \mathrm{~km}$ ) together with the assumed parent expansion velocity $\left(\sim 0.86 \mathrm{~km} \mathrm{~s}^{-1}\right)$ indicates that the lifetime of the $\mathrm{CN}$ parent at $1 \mathrm{AU}$ is in the order of $55000 \mathrm{~s}$, very close to the value deduced by Biver et al. (2000) for the inverse of the HCN photodissociation rate at $1 \mathrm{AU}\left(2.0 \times 10^{-5} \mathrm{~s}^{-1}\right)$ for a moderate to high solar activity. Moreover, the fact that the $Q_{\mathrm{CN}}$ presented here and the $Q_{\mathrm{HCN}}$ obtained by Mumma et al. (2001) are very similar seems to support the idea that the observed $\mathrm{CN}$ might arise only from $\mathrm{HCN}$, and that it is not necessary to invoke the presence of extended sources or other parent molecules beside HCN to explain the $\mathrm{CN}$ abundance in the coma of Comet Lee.

The water production rate derived in this work, $(1.22 \pm$ $0.70) \times 10^{29} \mathrm{~s}^{-1}$, can be considered as compatible with other published measurements from $\mathrm{OH}$ measurements at $18 \mathrm{~cm}$ and/or $3085 \AA$. Simultaneous to our optical observations, Comet Lee was observed by Biver et al. (2000) in the millimeter range as part of a campaign from May 4 to October 26, 1999. The $18 \mathrm{~cm} \mathrm{OH}$ lines in the comet were detected on a daily basis at the Nançay Radio Telescope. From 4 transits on 06/04.65-08.63 UT, Biver et al. (2000) deduced an average $\mathrm{OH}$ production rate of $(8.8 \pm 0.7) \times 10^{28} \mathrm{~s}^{-1}$. Assuming an $\mathrm{H}_{2} \mathrm{O} \rightarrow \mathrm{OH}$ branching ratio of $90 \%$, the $\mathrm{H}_{2} \mathrm{O}$ production rate is $1.1 \times Q_{\mathrm{OH}}$, that is $\sim 9.7 \times 10^{28} \mathrm{~s}^{-1}$. For the same date, Feldman et al. (1999) from the $\mathrm{OH}$ emission in the UV at $3085 \AA$ deduced a water production rate equal to $1.5 \times 10^{29} \mathrm{~s}^{-1}$, higher by $\sim 55 \%$. Considering the water production rate derived in this work, and that the $\mathrm{CN}$ is solely produced by $\mathrm{HCN}$ with a branching ratio of $100 \%$, we derive a value for $\mathrm{HCN} / \mathrm{H}_{2} \mathrm{O}$ of $0.25 \%$, slightly higher than the value deduced by Biver et al. (2000) from simultaneous observations of $\mathrm{OH}$ 
(and thus $\mathrm{H}_{2} \mathrm{O}$ ) and $\mathrm{HCN}$ (i.e. $0.11 \pm 0.02 \%$ ). Also, this $\mathrm{HCN}-$ to- $\mathrm{H}_{2} \mathrm{O}$ ratio is about the maximum found by Biver et al. (2002) from a sample of 24 comets studied at radio wavelengths, that were similar to the HCN enriched 109P/SwiftTuttle or C/1999 O1 (Hale-Bopp). These reported discrepancies $(0.25 \%$ versus $0.11 \%)$ may be due to several factors: (i) Biver et al. (2000) millimeter observations point to a lower gas activity, either in $\mathrm{H}_{2} \mathrm{O}$ or $\mathrm{HCN}$, and this is more noticeable in the case of $\mathrm{HCN}$ whose production rate is 3 times lower than the one obtained here from the $\mathrm{CN}$ red system, (ii) the uncertainties in the flux calibration of the spectra and in the $g$-factors of the $\mathrm{CN}$ red system, (iii) the systematic differences associated with the Haser modeling, and (iv) the well known discrepancy in the $\mathrm{H}_{2} \mathrm{O}$ water production rate when derived from the $\mathrm{OH} \mathrm{mm}$ and $18 \mathrm{~cm}$ lines, and $\mathrm{OH}$ in the $\mathrm{UV}$ or from $\mathrm{OI}\left({ }^{1} \mathrm{D}\right)$ emission at $6300 \AA$. Thus, $\mathrm{HCN} / \mathrm{H}_{2} \mathrm{O} \sim 0.27 \%$ with a $22 \%$ uncertainty. On the other hand, this hydrogen cyanide-to-water ratio drastically decreases if the Submillimeter Waver Astronomy Satellite (SWAS) measurements (Neufeld et al. 2000) are considered, as the $\mathrm{H}_{2} \mathrm{O}$ production rate is $50 \%$ higher with respect to Nançay data (radio observations of hydroxyl molecules).

The non-detection of the $\mathrm{CI}\left({ }^{1} \mathrm{D}\right)$ doublet at $9823 / 9850 \AA$ might arise from the fact that $\mathrm{C} / 1999 \mathrm{H} 1$ (Lee) shows a noticeable depletion in $\mathrm{CO}$ as compared to other long-period comets. More concisely, the $\mathrm{CO} / \mathrm{H}_{2} \mathrm{O}$ is 5-10 times lower than that expected for similar comets (Mumma et al. 2001). Biver et al. (2000) could only set an upper limit for the CO production on UT June $06.1, Q_{\mathrm{CO}}<5.60 \times 10^{27} \mathrm{~s}^{-1}$, whereas on UT August $24.8 Q_{\mathrm{CO}}=(2.6 \pm 0.8) \times 10^{27} \mathrm{~s}^{-1}$, meaning $\mathrm{CO} / \mathrm{H}_{2} \mathrm{O} \sim 1.8 \%$. By considering these production rates, $\mathrm{CI}\left({ }^{1} \mathrm{D}\right)$ column densities profiles can be computed and thus, the energy flux as a function of $\rho$. For a CO lifetime as the inverse of the photodissociation rate for high solar activity $\left(J=1.21 \times 10^{-6} \mathrm{~s}^{-1}\right)$, CO ejection velocity of $0.86 r_{h}^{-0.4} \mathrm{~km} \mathrm{~s}^{-1}, \mathrm{CI}\left({ }^{1} \mathrm{D}\right)$ excess velocity of $3.9 \mathrm{~km} \mathrm{~s}^{-1}$ (Huebner et al. 1992) and lifetime $\sim 4000 \mathrm{~s}$, the vectorial modeling (Festou 1981) predicts CI column densities ranging from $2.29 \times 10^{10} \mathrm{~cm}^{-2}$ and $<1.77 \times 10^{11} \mathrm{~cm}^{-2}$ at $\rho \sim 10000 \mathrm{~km}$, depending on the assumed $\mathrm{CO}$ production rate. Taking into account that the transition probability for the forbidden line ${ }^{1} \mathrm{D}_{2}-{ }^{3} \mathrm{P}_{2}(9850 \AA)$ within the ground state configuration of CI is $1.8 \times 10^{-4} \mathrm{~s}^{-1}$ (Czyzak \& Poirier 1985; Hibbert et al. 1993; Tozzi et al. 1998), these $\mathrm{CI}\left({ }^{1} \mathrm{D}\right)$ column densities would result into $1.7 \times 10^{-18} \mathrm{erg} \mathrm{cm}^{-2} \mathrm{~s}^{-1}$ and $<1.3 \times 10^{-17} \mathrm{erg} \mathrm{cm}^{-2} \mathrm{~s}^{-1}$, below the $3 \sigma$ flux upper limit measured in our observations. The CO production rate on June 6,1999 as derived from the $3 \sigma$ upper limit of CI infrared doublet, is $\leq 5.75 \times 10^{28} \mathrm{~s}^{-1}$, an order of magnitude higher than previous determinations by Biver et al. (2000).

Spectroscopic observations of $\mathrm{NH}_{2}$ give hints on the ammonia abundance in comets. The photolytic reaction for photodissociation of ammonia $\mathrm{NH}_{3}+h v \rightarrow \mathrm{NH}_{2}+\mathrm{H}$ has a $95 \%$ quantum yield. Therefore, provided that $\mathrm{NH}_{3}$ is the dominant source of $\mathrm{NH}_{2}$, the abundance of the latter is essentially a direct measurement of the ammonia abundance in comets. However, as Meier et al. (1994) have noted, the $\mathrm{NH}_{3} / \mathrm{H}_{2} \mathrm{O}$ deduced from spectroscopic measurements of $\mathrm{NH}_{2}$ gives systematically very low, i.e. in a factor of $\sim 2$, ammonia-to-water ratios or present considerable scatter. The ammonia measurements carried out in situ for comet $\mathrm{P} /$ Halley by the Giotto spacecraft indicate that this discrepancy can be as high as one order of magnitude. The ammonia-to-water abundance for Comet Lee ranges from $\sim 1 \%$ to $\sim 4 \%$ depending on the considered $g$-factors, viz. those given by Kawakita \& Watanabe (2000) or Tegler \& Wyckoff (1989) corrected according to Arpigny (1995), respectively. This ammonia-to-water ratio is slightly higher than the $Q_{\mathrm{NH}_{3}} / Q_{\mathrm{H}_{2} \mathrm{O}}$ reported for other long-period comets, either old or dynamically new (see Feldman et al. 1993), placing the ratio between $0.4 \%$ and $0.8 \%$ within a factor of 2 , and more recently for Comet Hyakutake (0.3\%) derived by Palmer et al. (1996), whereas it is closer to the estimates in Meier et al. (1994) from the Giotto flyby measurements and to the predictions by gas coma photochemical models (Allen et al. 1987).

Our estimate of the gas-to-dust mass ratio indicates that C/1999 H1 can be considered as a relatively dust poor comet. The behaviour of the dust grains, as deduced from the study of the $\log B$ vs. $\log \rho$ points to a steady-state and isotropic outflow of the solid cometary component composed of long-lived grains. The quantitative composition and size distribution of these grains can be inferred from Mie-scattering modeling. The observed spectrum in the range $6000-10000 \AA$ can be fitted by a dust grain population of silicates and icy grains whose mean radius is $\sim 1 \mu \mathrm{m}$. Some discrepancies between observations and modeling are seen beyond $8700 \AA$ likely due to the use of constant (i.e. non-wavelength dependent) refractive index of the dust material. For our spectroscopic range, the real part of the complex refractive index for representative cometary material can be considered constant with wavelength, whereas the imaginary part shows noticeable variations not taken into account in our computations. Regardless of what the continuum bandpass we consider, the color of the dust is almost constant at every $\rho$ in east and west direction, only showing a steady reddening at increasing $\rho$ from the nucleus in both directions. At distances higher than $25000 \mathrm{~km}$, the differences between the east and west direction are likely due to the approximate 2D sky subtraction and not to real east-west differences in the dust population.

Although Comet Lee was not extensively monitored, the available observations of this comet have provided us with a new opportunity for studying the behaviour of another long-period comet lately visiting the inner Solar System. From a general point of view, Comet Lee, C/1999 H1 can be considered as a rather depleted $\mathrm{CO}$ comet, whereas it is considerably abundant in $\mathrm{CH}_{3} \mathrm{OH}$ according to Biver et al. (2000). In this respect, comet $\mathrm{C} / 1999 \mathrm{H} 1$ is intrinsically different from C/1995 O1 (Hale-Bopp) and C/1996 B2 (Hyakutake), although the three comets are thought to have a common origin. Regarding carbon-chain species, the abundance of ethane and acetylene with respect to that of water, $(\sim 0.6$ and $\sim 0.3$, respectively) is much the same for the three comets (Mumma et al. 2001). The optical data obtained at ESO also allow us to compare Comet Lee with some of the comets presented in the surveys by A'Hearn et al. (1995) and Fink \& Hicks (1996). Comparison of the $\log$ of the production rates ratios $\frac{\mathrm{CN}}{\mathrm{OH}}$ and $\frac{A f \rho}{\mathrm{OH}}$ for comet $\mathrm{C} / 1999 \mathrm{H} 1$ with the values for the restricted data set in Table 4 of A'Hearn et al. (1995) indicates that Comet Lee is similar to periodic comets 23P/Brorsen-Metcalf, 
$6 \mathrm{P} / \mathrm{d}$ 'Arrest or $2 \mathrm{P} /$ Encke and to long period comets such as Okazaki-Levy-Rudenko (C/1989 Q1), Thiele (C/1985 T1) or Sugano-Saigusa-Fujigawa (C/1983 J1). It should be noted that the previously mentioned comets all bear the characteristic of being "typical" within the taxonomy introduced by A'Hearn et al. (1995), that is, $\log \frac{Q\left(\mathrm{C}_{2}\right)}{Q(\mathrm{CN})} \geq-0.18$, and for all of them this ratio lies between -0.09 and 0.29 . This characteristc also seems to be present in Comet Lee as $\log \frac{Q\left(\mathrm{C}_{2}\right)}{Q(\mathrm{CN})} \sim 0.28$, that is, the abundance of carbon-chain species $\left(\mathrm{C}_{2}\right)$ can be considered as typical, assuming that either acetylene or ethane are the only precursors of $\mathrm{C}_{2}$ with $100 \%$ efficiency.

Similarly, of the 39 comets surveyed by Fink \& Hicks (1996), comet C/1999 H1 resembles comets $73 \mathrm{P} /$ Schwassmann-Wachmann 3 or $2 \mathrm{P} /$ Encke in terms of $\mathrm{CN}$ to $\mathrm{H}_{2} \mathrm{O}$ relative abundance. Comparison of the $\mathrm{NH}_{2}$ content versus $\mathrm{H}_{2} \mathrm{O}$ in the same database is more uncertain given the new results on the $g$-factors by Kawakita \& Watanabe (2002). Previous studies (Fink 1994; Fink \& Hicks 1996; Hicks \& Fink 1997; Fink et al. 1999) showed that there is some dependency on the heliocentric distance for the $\mathrm{NH}_{2}$ abundance; namely, the $\mathrm{NH}_{2}-$ to- $\mathrm{H}_{2} \mathrm{O}$ ratio becomes larger for larger $r_{h}$. The new determination of the $\mathrm{NH}_{2}$ fluorescence efficiency (as a function of $r_{h}$ ) removes this dependency, giving rise to $\mathrm{NH}_{2}$-to- $\mathrm{H}_{2} \mathrm{O}$ ratios nearly constant within the range from 0.5 to $3 \mathrm{AU}$. Basing our comparison on the $\mathrm{NH}_{2}$ production rates derived by means of the most recent $g$-factors (i.e. Kawakita \& Watanabe 2002), the $\mathrm{NH}_{2} / \mathrm{H}_{2} \mathrm{O}$, and thus ammonia-to-water ratio, in comet $\mathrm{C} / 1999 \mathrm{H} 1$ (Lee) is $\sim 1 \%$, consistent with what was found for 1P/Halley, C/1996 B2 (Hyakutake) and C/1996 O1 (Hale-Bopp), that is, $0.5 \%, 0.6 \%$ and $1.5 \%$, respectively.

\section{Summary}

Long-slit spectrophotometric observations of Comet Lee (C/1999 H1) were acquired on June 6, 1999. For slit acquisition and pointing purposes, several comet images were obtained with $R$ Bessel and Gunn $i$ filters. The morphological anaylsis of these images has unveiled the presence of two pairs of ion rays slowly converging to the tail axis during the $45 \mathrm{~min}$ the comet was imaged. As the comet was not observed in interference filters, the existence of dust and/or neutral gas structures cannot be ascertained.

$\mathrm{CN}$ and $\mathrm{NH}_{2}$ column densities have been derived from the long-slit spectra. The $Q_{\mathrm{CN}}$ and $Q_{\mathrm{NH}_{2}}$ production rates have been obtained by means of the Haser model yielding to $\sim 3 \times 10^{26} \mathrm{~s}^{-1}$ and $\sim 1 \times 10^{27} \mathrm{~s}^{-1}$, respectively. The water production rate $Q_{\mathrm{H}_{2} \mathrm{O}} \sim 1.22 \times 10^{29} \mathrm{~s}^{-1}$, inferred from the luminosity of the $\mathrm{OI}\left({ }^{1} \mathrm{D}\right)$ line at $6300 \AA$ is in reasonable agreement with values reported by Biver et al. (2000) and by Feldman et al. (1999) on the same date. The $\mathrm{CI}\left({ }^{1} \mathrm{D}\right)$ doublet at $9823 / 9850 \AA$, indicative of the CO abundance in $\mathrm{C} / 1999 \mathrm{H} 1$, has not been undoubtedly detected, only $3 \sigma$ upper limit flux emission $\sim 4 \times$ $10^{-17} \mathrm{erg} \mathrm{cm}^{-2} \mathrm{~s}^{-1}$, that is $Q_{\mathrm{CO}}<5.78 \times 10^{28} \mathrm{~s}^{-1}$, has been inferred. The ratios of $\mathrm{HCN}$ and $\mathrm{NH}_{3}$ to $\mathrm{H}_{2} \mathrm{O}$ are $\sim 0.28 \%$ and $\sim 1 \%$, in agreement with what has been obtained for other longperiod comets.

The Af $\rho$ parameter, a measure related to the dust production rate, is relatively low $(\sim 500 \mathrm{~cm})$ allowing us to classify this comet as a poor dust comet with a gas-to-dust mass ratio in the range between 6 and 12 depending on the measurements that provide the $\mathrm{OH}$ (and thus, water) production rate. The dust brightness profiles in east-west direction do not deviate from the canonical law $\log B \sim m \log \rho$ with $m=1.01 \pm 0.16$, whereas the dust slightly reddens with distance from the nucleus. The dust grain population can be characterised by a relatively broad size distribution whose solid particles have a quite common mean radius of $1 \mu \mathrm{m}$ and are composed of a mixture of silicates and icy grains.

Although the organic volatile composition of the long period comet C/1999 H1 (Lee) very much resembles that of other long period comets (such as Hyakutake or Hale-Bopp) pointing to a common origin and/or evolution, other peculiarities are also present in some Jupiter family comets such as 2P/Encke, $6 \mathrm{P} / \mathrm{d}$ 'Arrest or $73 \mathrm{P} / \mathrm{Sch}$ wassmann-Wachmann 3, or in $1 \mathrm{P} /$ Halley, a long-period and dynamically old comet. For a more meaningful characterisation of comet Lee, a wider covering of its behaviour as a function of the heliocentric distance would have been desirable. However, given the available information and in the current state, Jupiter family comets with likely origin in the Kuiper Belt, Halley-type comets and longperiod comets presumably coming from the Oort Cloud do not show clearly distinct compositions.

Acknowledgements. L.M.L is grateful to G. H. Jones for fruitful conversations on ion rays. The research carried out has been partially supported by the Spanish Ministerio de Ciencia y Tecnología under contracts PNE-002/2000-C and PNE-001/2000-C-01.

\section{References}

A'Hearn, M. F., Schleicher, D. G., Millis, R. L., Feldman, P. D., \& Thompson, D. T. 1984, AJ, 89, 579

A'Hearn, M. F., Hoban, S., Birch, P. V., et al. 1986, Nature, 324, 649

A'Hearn, M. F., Millis, R. L., Schleicher, et al. 1995, Icarus, 118, 223

Allen, M., Delitsky, M., Huntress, W., et al. 1987, A\&A, 187, 502

Arpigny, C., Magain, P., Manfroid, J., et al. 1987a, AJ, 187, 485

Arpigny, C., Manfroid, J., Magain, P., et al. 1987b, ESA SP-278, 571

Arpigny, C. 1995, ASP Conf. Ser., 177, 71

Biver, N., Bocklée-Morvan, D., Crovisier, J., et al. 2000, AJ, 120, 1554

Boehnhardt, H., \& Birkle, K. 1994, A\&A, 107, 101

Bohren, C. F., \& Hufmann, D. R. 1983, in Absorption and scattering of light by small particles (New York: Wiley)

Budzien, S. A., Festou, M. C., \& Feldman, P. D. 1994, Icarus, 107, 164

Chiu, K., Neufeld, D. A., Bergin, E. A., et al. 2001, Icarus, 154, 345

Combi, M. R., \& Delsemme, A. H. 1980, ApJ, 237, 633

Combi, M. R., \& McCrosky, R. E. 1991, Icarus, 91, 270

Czyzak, S. J., \& Poirier, C. P. 1985, Ap\&SS, 116, 21

Feldman, P., Fournier, K. B., Grinin, V. P., \& Zvereva, A. M. 1993, ApJ, 404, 348

Feldman, P. D., Weaver, H. A., A'Hearn, M. F., et al. 1999, BAAS, 31, 1127

Festou, M. C. 1981, A\&A, 95, 69

Festou, M., \& Feldman, P. 1981, A\&A, 103, 154

Fink, U. 1994, ApJ, 423, 461

Fink, U., \& Hicks, M. D. 1996, ApJ, 459, 729

Fink, U., Hicks, M. D., \& Fevig, R. A. 1999, Icarus, 141, 331

Hansen, J. E., \& Travis, L. D. 1974, Space Sci. Rev., 16, 527

Haser, L. 1957, Bull. Cl. Sci. Acad. R. Belg., 43, 740 
Hibbert, A., Biemont, E., Godefroid, M., \& Vaeck, N. 1993, A\&AS, 99, 179

Hicks, M. D., \& Fink, U. 1997, Icarus, 127, 307

Melnick, G. J., Gary, J., Stauffer, J. R., et al. 2000, ApJ, 539, L77

Mumma, M. J., McLean, I. S., DiSanti, M. A., et al. 2001, ApJ, 546, 1183

Huebner, W. F., Keady, J. J., \& Lyon, S. P. 1992, Adv. Space Sci., Neufeld, D. A., Stauffer, J. R., Bergin, E. A., et al. 2000, ApJ, 539, 195,1

Huebner, W. F., \& Link, R. 1999, http://espsun.space.swri.edu/spacephysics/ atomic/html/photoabs.htm

Kawakita, H., \& Watanabe, J. 2002, ApJ, 572, L177

Kolokolova, L., Lara, L. M., Schulz, R., Stüwe, J. A., \& Tozzi, G. P. 2001, Icarus, 153, 197

Kurucz, R. L., Furenlid, I., Brault, J., \& Testerman, L. 1984, National Solar Observatory Atlas, No. 1, June 1984

Labs, D., Neckel, H., Simon, P. C., \& Thuiller, G. 1987, Sol. Phys., 90, 25

Meier, R., Eberhardt, P., Krankowsky, D., \& Hodges, R. R. 1994, A\&A, 287, 268 